\title{
Short Commentary: More Health Psychology Intervention Needed to Prevent Traumatic Brain Injuries
}

\section{Kathy Sexton-Radek}

Elmhurst College, Suburban Pulmonary and Sleep Associates, Westmont, USA

*Corresponding author: Kathy Sexton-Radek, Elmhurst College, Suburban Pulmonary and Sleep Associates, Westmont, USA, Tel: +6306173587; E-mail: kathysr@elmhurst.edu

Received date: February 01, 2018; Accepted date: February 08, 2018; Published date: February 14, 2018

Copyright: () 2018 Sexton-Radek K. This is an open-access article distributed under the terms of the Creative Commons Attribution License, which permits unrestricted use, distribution, and reproduction in any medium, provided the original author and source are credited.

\section{Commentary}

As Health Care Professionals, we are familiar with the devastating outcomes of Traumatic Brain Injury (TBI). Findings indicated that some 16.4 per 100, 0001 individuals sustain a TBI annually. The TBI injuries are from a variety of impact sources where mechanical forces cause the initial injury and the subsequent sequelae.

It is the sequelae that present a complication for the patient and medical care as the level of awareness regarding their significance is commonly underestimated. With TBI, it is probable that severe medical consequences are complicated further by the absence of serious signs of brain tissue damage at initial examinations. As a result, many are treated and released and a greater number do not seek medical care [1].

TBI are categorized from mild to severe depending on the degree of impairment. The mild TBI are most often seen by a Medical professional and with the absence of symptomology, discharged for observation and further care if the need arises.

The situation is further complicated by psychological factors reduced cognitive ability in terms of attention and concentration and emotional deregulation in terms of irritability and impulsivity [2]. Brain imaging and laboratory tests such as the S100 biomarker offer some objective measurements of TBI; however, statistics indicate little use within the context of the number of TBI cases [3].

This contemporary situation of increased incidence of TBI is not new for Health Psychology research and practice consideration. The actions of initial impact and rotation with helmet use have been well studied [2]. Both linear and rotational head accelerations after an impact have been investigated in helmet studies.

Additionally, the impact locations on the brain and subsequent shearing of brain tissue have been investigated with helmet use [2]. Also, the restraint of a driver with a seat belt has been investigated. Because of empirical findings from a myriad of Health Psychology studies, mandatory seat belt and universal helmet use laws in most states were enacted in the 1980s and 1990s, respectively.

There are additional identified factors that contribute to TBI. Such factors as fall prevention, pedestrian safety and anonymous reporting are of shaken baby syndrome are promoted as education and intervention prevention strategies to abate TBI incidence figures. Everyday circumstances such as falls and walking near traffic sites have been identified in the descriptive literature but little data exist in the effectiveness of such interventions.

The anonymous reporting of Shaken Baby syndrome by Departments of Children and Family Services is designed for immediate, private reporting's; research reporting's of the frequencies of reporting's are not published. The resultant post concussive symptoms (e.g., cognitive attention and concentration decline, emotional labiality depressed mood, irritability) often occurring in TBI.

However, severity of TBI symptoms-from impact or post-impact have not been found consistently related. Individual differences in reporting's of TBI symptoms, coping level, social support levels have been identified as potential modifiers of reporting TBI symptoms [1,2]. Despite this, it is hoped but not substantiated that the severe consequences in functioning and human suffering from TBIs is reduced with prevention strategies [2].

Increasing reporting's of TBIs and emerging findings from neuroscience of the injuries and concomitant brain tissue damage escalates the need to focus on the measurement of interventions. Given that prevention is paramount to addressing TBIs, a clear understanding of the effectiveness of our interventions and resultant thinking of further prevention means is essential.

With the incidence rates of TBIs identifying children and primarily young adults being afflicted [3], perhaps an extension of the conventional means of prevention messaging (e.g., public service announcements, information in schools and medical facilities, media) could be expanded to the modality this population uses-the internet. Social media messaging and perhaps an app to check if you have signs of poor functioning and need to contact the nearest medical professional may work [4].

In general, the context in which the TBI occurs needs further assessment. The study of sports and events during each sport that are highly related to potential head impact need to be studied.

Further, the messaging form media, medical and legal information conveyed to patients could be examined to determine how it more fully impact the patient's/potential patient of TBI consequences. With these efforts, further understanding of the at-risk conditions and at-risk groups for TBI can be determined. Health care promotion by Health Care professionals and researchers are best suited to expand our prevention efforts addressing TBIs with research agendas and direct preventive information to the at-risk groups.

\section{References}

1. Indiana State Department of Health (2017) Special Emphasis Report: Traumatic Brain Injury, 2015. Division of Trauma and Injury Prevention.

2. Bazarian JJ, Cernak I, Noble-Haeusslein L, Potolicchio S, Temkin N (2009) Long-term neurologic outcomes after traumatic brain injury. Journal of Head Trauma Rehabilitation 24: 439-451.

3. Roebuck-Spencer T, Sherer M (2018) Moderate and Severe Traumatic Brain Injury, pp: 387-410. 
Citation: Sexton-Radek K (2018) Short Commentary: More Health Psychology Intervention Needed to Prevent Traumatic Brain Injuries. J Health Educ Res Dev 6: 249. doi:10.4172/2380-5439.1000249

Page 2 of 2

4. Morgan JE, Ricker JH (2018) Textbook of Clinical Neuropsychology. 2nd edn. New York: Routledge Taylor \& Francis Group Publishers. 